\title{
Sonographic Abnormalities of Thyroid Gland in Post- Menopausal Women with and without Hormone Replacement Therapy
}

\author{
Namra Adnan $^{1 *}$, Syeda Khadija-Tul-Sughra Murrium ${ }^{2}$, Syed Amir Gilani ${ }^{3}$ \\ ${ }^{1}$ MMUT (ultrasound), MIT, Lahore, Pakisthan. \\ ${ }^{2}$ MSDU (Ultrasound), Assistant professor Department (UIRSMIT) FAHS, Lahore, Pakisthan. \\ ${ }^{3}$ Dean FAHS, University of Lahore, Pakisthan.
}

*Corresponding Author: Namra Adnan, MMUT (ultrasound), MIT, Lahore, Pakisthan.

Received Date: January 17, 2020; Accepted Date: March 15, 2021; Published Date: March 17, 2021.

Citation: N Adnan, Syeda K-T-S Murrium, Syed A Gilani. (2021) Sonographic abnormalities of thyroid gland in post-menopausal women with and without hormone replacement therapy. Journal of Endocrinology and Disorders. 5(2): Doi: 10.31579/2640-1045/061

Copyright: (C) 2021 Namra Adnan. This is an open-access article distributed under the terms of the Creative Commons Attribution License, which permits unrestricted use, distribution, and reproduction in any medium, provided the original author and source are credited.

\begin{abstract}
:
Background: Thyroid dysfunction is common especially in women over the age of 50 years. The prevalence of thyroid abnormalities is more in postmenopausal women using hormone replacement therapy. The prevalence of biochemical (or subclinical) hypothyroidism in women increases steadily with age, rising from $10 \%$ to $20 \%$ in the postmenopausal. The study results clearly indicate an increase in the activities of TSH among the older-aged post-menopausal women.

Objective: To compare the sonographic findings of thyroid gland in post-menopausal women with and without hormone replacement therapy.

Material and Method: A review of scientific literature concerning the correlation of Sonographic abnormalities of thyroid gland in post-menopausal women with and without hormone replacement therapy was done. Different platforms including medical journals, books and online resources(e.g., PubMed, Google Scholar \& Medline) were explored to find the relevant data using the mesh Terms: Thyroid gland, postmenopausal women, Hormone Replacement Therapy etc. The main focus was given to the latest data published in the last 10 years.
\end{abstract}

Key words: Thyroid gland, postmenopausal women, Hormone Replacement Therapy.

\section{Introduction:}

Hormone Replacement Therapy is a type of hormone therapy used to treat various symptoms associated menopause among females. The main hormones used in HRT are estrogens and progesterone. Estrogen has a well-known indirect effect on thyroid economy, increasing the thyroxine binding globulin, the need for thyroid hormone in hypothyroid women [1]. HT is indicated for the treatment of postmenopausal women with intact uteri, whereas ET is used in women who have had hysterectomies [2].Incidence of most thyroid diseases: hypothyroidism, nodular goiter, and cancer is highest among postmenopausal and elderly women. The diagnosis of thyroid dysfunction in this group of patients is difficult because the symptoms can be nonspecific or common with menopausal and ageing complaints. Thyroid nodules and cancer often affect women over 50 years old; the diagnostic and therapeutic approach is the same as in the general population, but the surgical risk and cancer prognosis is worse than in young patients [3].

\section{Literature Review:}

The thyroid is a butterfly-shaped gland that sits low on the front of the neck. Thyroid lies below Adam's apple, along the front of the windpipe. The thyroid has two side lobes, connected by a bridge (isthmus) in the middle. The thyroid secretes several hormones, collectively called thyroid hormones. The main hormone is thyroxine, also called T4. The arterial supply to the thyroid gland is via two main arteries: Superior thyroid artery, Inferior thyroid artery. Ultrasound (US) investigations are commonly performed with a 4-cm-long linear transducer. Threedimensional imaging can demonstrate additional planes, such as the coronal carotid thyroid plane. The transducer with a medium frequency of $7.5 \mathrm{MHz}$ represents a good compromise between resolution and penetration. Large goiters, however, can only be delineated with frequencies of $5 \mathrm{MHz}$ or lower. For very superficial lesions or infiltrations in the strap muscles frequencies of $10 \mathrm{MHz}$ or more are used [6]. 
Hormone replacement therapy (HRT) is a form of hormone therapy used to treat symptoms associated with female menopause. An increased trend of HRT by postmenopausal women has been reported over the past decade and now women more encouraged to choose hormone replacement therapy. Considerable data available on the role of HRT in relieving menopausal symptoms i.e. hot flushes, vaginal atrophy and dryness, insomnia and in preventing osteoporosis and urogenital atrophy. HRT is not only beneficial in improving menopausal symptoms but it also helps in preventing and managing osteoporosis [7, 8]. This study is conducted by SOLOMON BL, in 1993. We interviewed 300 white postmenopausal women (160 with thyroid disease, 140 without thyroid disease) to investigate whether having thyroid disease or taking thyroid hormone increased the prevalence of having a hip, vertebral, or forearm fracture. Thirty-seven (23\%) women with thyroid disease and 45 (32\%) women without thyroid disease had had a fracture, and there were no significant differences between these groups in the number or type of fractures. Dose of thyroid hormone and duration of therapy or disease did not affect fracture occurrence in women with thyroid disease. Women with a history of hyperthyroidism ( 9 of 32 ) or thyroid cancer ( 2 of 11) appeared to have their first fracture earlier $(p<0.01)$ than women without thyroid disease. In summary, women taking thyroid hormone for a variety of thyroid disorders do not appear to have an enhanced prevalence of a hip, vertebral, or forearm fractures, but women with a history of hyperthyroidism may have a propensity for their fractures to occur earlier in life [9]. This study was conducted by Del Ghianda $S$ in 2018, thyroid dysfunction is common in the general population especially in women. All thyroid diseases are in fact more common in women than in men and may interfere with the reproductive system. Thyroid function and the gonadal axes are related throughout the woman's fertile period. The relationship between the two glands is mutual. In particular, thyroid hormones affect the reproductive function both directly and indirectly through several actions. Studies on the relationship between menopause and thyroid function are few and do not allow to clarify whether menopause has an effect on the thyroid regardless of aging. With aging, the main changes regarding thyroid physiology and function are: a reduction of thyroid iodine uptake, free thyroxine and free triiodothyronine synthesis and catabolism of free thyroxine while reverse triiodothyronine increases; the level of thyroid stimulating hormone remains normal with sometimes a tendency to higher limits. These changes are present in both sexes without distinction between males and females. The complexity of the relationships can be summarized in three aspects: thyroid status does not influence significantly the climacteric syndrome; menopause may modify the clinical expression of some thyroid diseases, particularly the autoimmune ones; thyroid function is not directly involved in the pathogenesis of the complications of menopause. However, coronary atherosclerosis and osteoporosis may be aggravated in the presence of hyperthyroidism or hypothyroidism. The effects of postmenopausal estrogen replacement on thyroxine requirements in women with hypothyroidism should be considered [10]. In 2018 jan 1, Rubio GA study was conducted on , Estrogen receptors can regulate growth in papillary thyroid cancer and may affect prognosis after menopause. This study examines changes of estrogen receptor subtype ratio expression in papillary thyroid cancer cell lines derived from preand postmenopausal women [12]. Solbiati L, in 1985 Radionuclide studies have, for many years, been the most commonly used imaging method in the evaluation of nodular abnormalities of the thyroid gland. Despite its leading role, scintigraphy has proved to be unsatisfactory in assessing the nature of the hypo functioning thyroid even with the introduction of labeled test agents. At present ultrasonography (US) plays an outstanding role in the study of isotopically cold lesions (4-10), but, to our knowledge, no report based on large groups of patients has been published in which the specific echognaphic patterns of the various pathologic processes are defined. Such findings will be analyzed in this paper, which is based on a large series of surgically and pathologically proved cases studied at several institutions. The incidence of the various abnormalities in the population will be appraised as well [13].

In 2019, Bhagavan Reddy Kolanu conducted the study results clearly indicate an increase in the activities of TSH among the older-aged postmenopausal women. Autopsy studies have reported incidental thyroid nodules in up to $50 \%$ of subjects. Most nodules are benign, but between 3 and 7\% of cases are found to be malignant [14] Rallison ML, in 1991 thyroid diseases, including Graves' disease, nontoxic goiter, Hashimoto's thyroiditis, and thyroid neoplasms, occur more frequently in women than in men, and their natural history has been described in adults . Comparable observations on the prevalence and natural history of thyroid disease in children and adolescents are fragmentary and largely based on studies of selected clinic populations rather than on studies of a general population. In an area of the southwestern United States exposed to radiation in fallout from testing of nuclear devices, we screened a population of young persons for thyroid disease and followed them over a period of 20 years, from adolescence to their mid-30s. On the basis of our observations of the thyroid in this cohort, the prevalence, incidence, regression, and natural history of thyroid abnormalities (simple goiter, thyroiditis, and thyroid nodularity, including benign and malignant neoplasms) in a young population living in the western U.S. were determined [15]. Ceresini G, conducted this study in 2008, Estrogen receptors are present in thyroid follicular cells in normal and neoplastic tissue. We evaluated changes in total thyroid volume and volume of thyroid nodules in postmenopausal women given either hormone therapy (HT) or no treatment in a 1-year observational follow-up [16].

Sajjadieh HR, in 2005 Thyroid nodules are common and may be caused by a variety of thyroid disorders. The prevalence of palpable thyroid nodules in members of the general population who are screened by palpation is $1 \%$ to $7 \% .1$ Although most are benign, about $5 \%$ to $10 \%$ of all palpable nodules are malignant.2-5 Many tests and procedures are available for evaluating thyroid nodules, and appropriate selection of tests is important for accurate diagnosis. Sonography was performed by one of two radiologists at Khorshid Hospital, Esfahan, on 134 patients with suspected thyroid nodules. Thyroid nodules were found in 100 of 134 patients by sonography; the remaining 34 were excluded from the study group. A real-time scanner equipped with a $7.5-\mathrm{MHz}$ linear transducer (EUB 40, Hitachi) was used in all sonographic examinations. The second box of the checklist in Figure 1 was completed by the sonographers. In patients with more than one nodule, sonographic characteristics were evaluated on the dominant (palpable) and/or suspected malignant nodule [17]. 

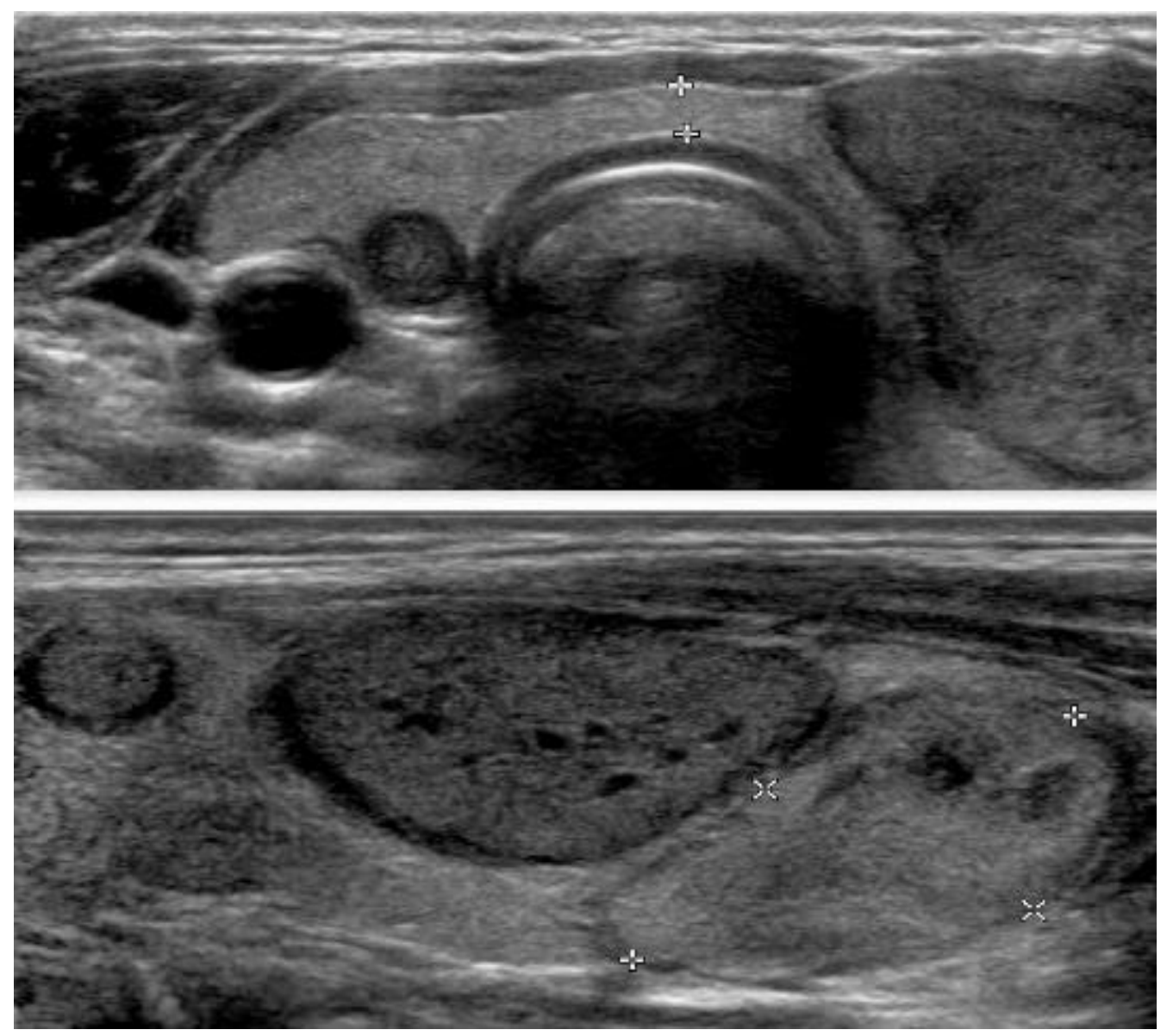

Figure 1: Transverse ultrasound image of the thyroid gland shows the single nodule in the right lobe. Longitudinal ultrasound image of the left lobe of the thyroid shows the three nodules in the left lobe of the thyroid [4].
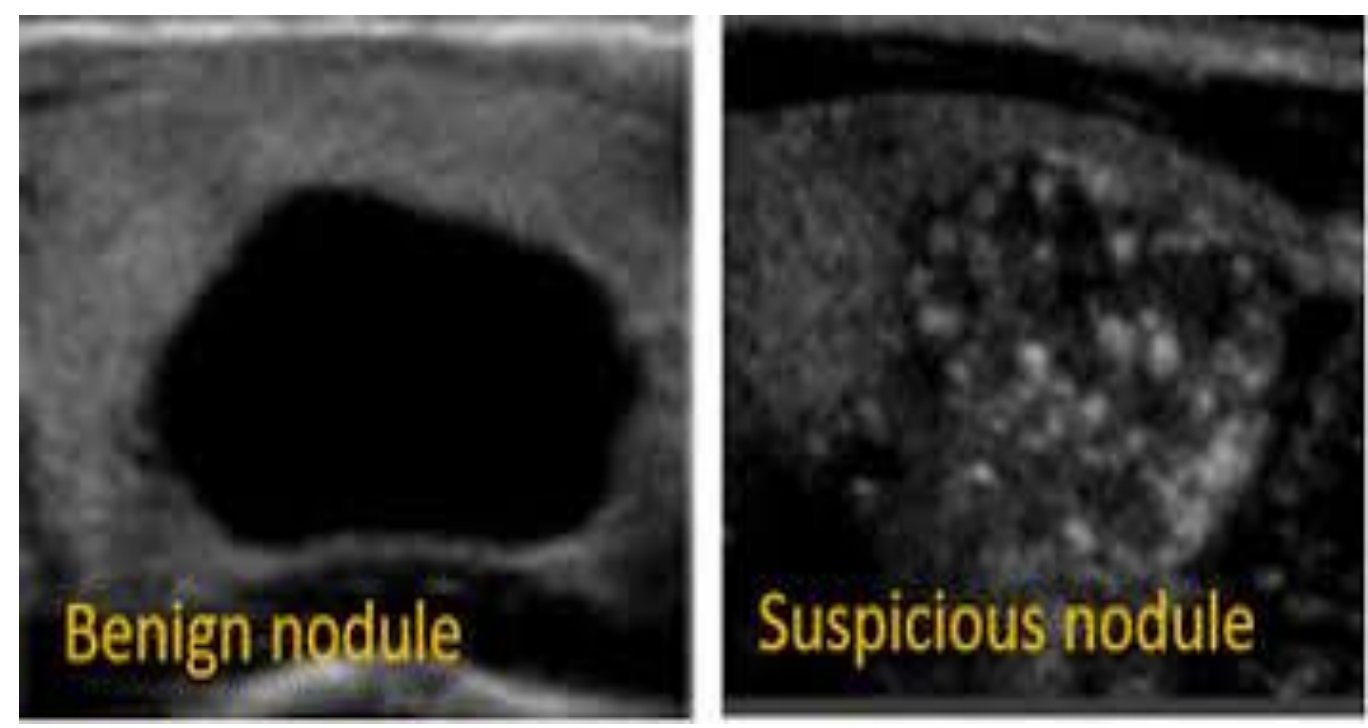

Figure 2a: shows thyroid nodules that appear purely cystic, or filled with fluid. 2b: Nodules that do not have smooth borders or have little bright white spots (micro-calcifications) on the ultrasound would make suspicious that there is a thyroid cancer present [5]. 

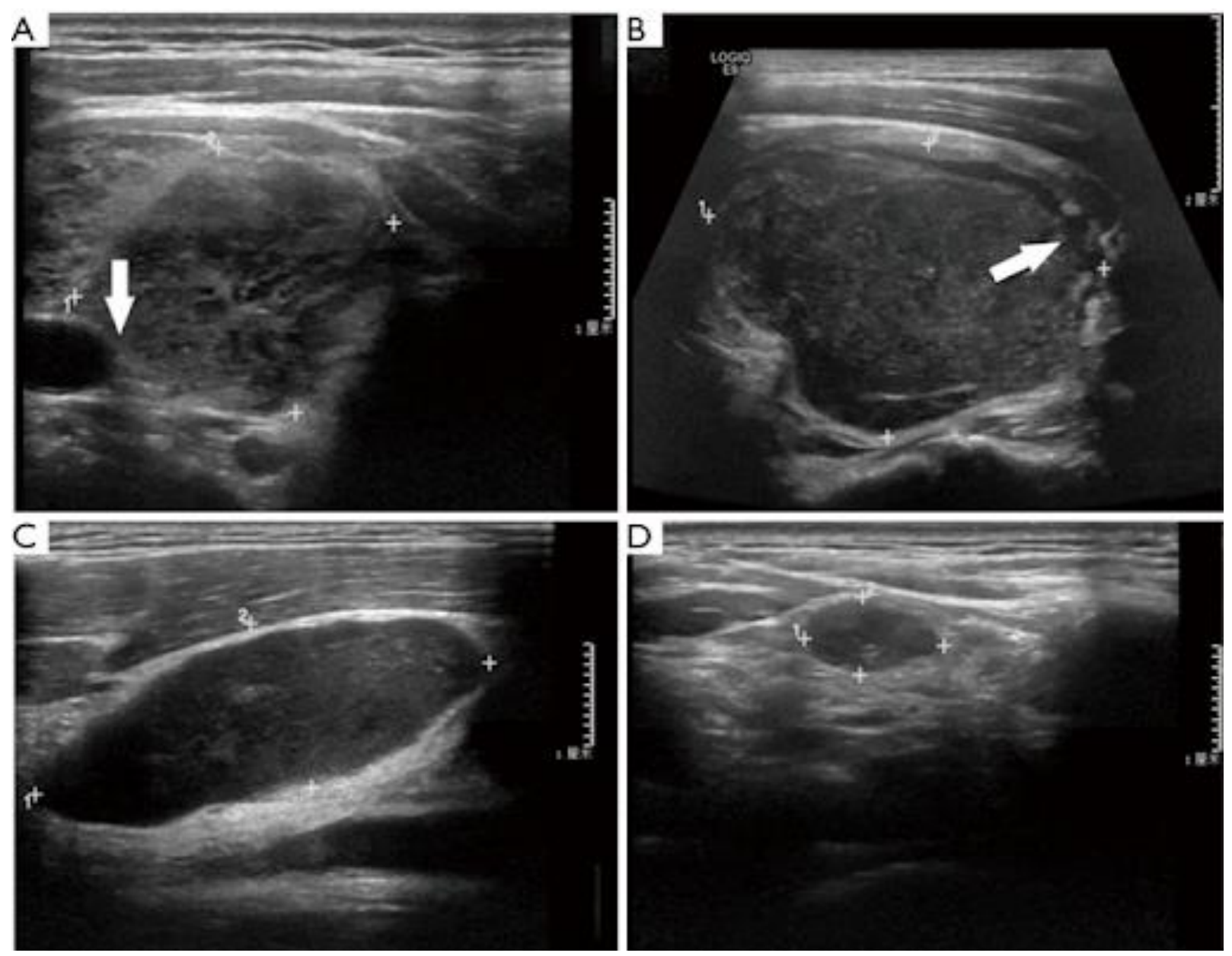

Figure 3a: Transverse image of ultrasonography showed nodular type of PTL. There was an irregular-shaped hypoechoic mass in the right lobe of the thyroid. The lesion had invaded the vascular wall,3b transverse image of ultrasonography showed a markedly hypoechoic mass in the left lobe of the thyroid with invasion of thyroid capsule.3c,3d longitudinal image showed pathologically confirmed involved lymph nodes in the left cervical III region appeared as markedly hypoechoic lymph nodes lacking echogenic hilum [11].

\section{Discussion:}

A thyroid nodule is solid or liquid mass within the thyroid. These nodules are frequently observed in normal healthy population. It is a common reported finding on ultrasonography. Incidental findings of thyroid nodules are being reported in about $50 \%$ population. Mostly nodules are benign, but $3 \%$ to $7 \%$ cases are reported to be malignant. Use of estrogen therapy/hormone therapy in postmenopausal women and the prevalence of hypothyroidism in this population, it is estimated that approximately $5 \%$ of all postmenopausal women receive treatment with both ET/ HT and thyroid hormone replacement. The issue of body weight has a great importance regarding the compliance to the postmenopausal hormonal replacement therapy. Premenopausal women commonly correlate the increase in body weight with hormone use. Conversely, different lines of evidence suggest that menopause is related to an increase in body weight and to an android body fat distribution. In this prospective study we further evaluated the pattern of body weight and body fat distribution in the early postmenopausal period in women with and without HRT administration.

\section{Conclusion:}

Thyroid diseases are more common in postmenopausal women than in premenopausal. Postmenopausal Females who are on ET/HRT have high incidence of thyroid diseases.

\section{References:}

1. Mazer NA. (2004) Interaction of estrogen therapy and thyroid hormone replacement in postmenopausal women. Thyroid. 14(3, Supplement 1):27-34.

2. Gietka-Czernel M. (2017) The thyroid gland in postmenopausal women: physiology and diseases. Przeglad menopauzalny= Menopause review. 16(2):33.

3. https://pediatriceducation.org//06/07/how-do-you-evaluateand-treat-a-thyroid-nodule/.

4. https://www.uclahealth.org/endocrine-center/thyroid-nodules.

5. Gritzmann N, Koischwitz D, Rettenbacher T. (2000) Sonography of the thyroid and parathyroid glands. Radiologic Clinics. 38(5):1131-45.

6. Rymer J, Wilson R, Ballard K.(2003) Making decisions about hormone replacement therapy. Bmj. 326(7384):322-6.

7. Ceresini G, Milli B, Morganti S, Maggio M, Bacchi-Modena A, Sgarabotto MP, Chirico C, Di Donato P, Campanati P, Valcavi R, Ceda GP.( 2008) Effect of estrogen therapy for 1 year on thyroid volume and thyroid nodules in postmenopausal women. Menopause. 15(2):326-31.

8. SOLOMON BL, WARTOFSKY L, BURMAN KD. (1993) Prevalence of fractures in postmenopausal women with thyroid disease. Thyroid. 3(1):17-23. 
9. Del Ghianda S, Tonacchera M, Vitti P. (2014) Thyroid and menopause. Climacteric. 17(3):225-34.

10. http://jtd.amegroups.com/article/view/15862/html.

11. Rubio GA, Catanuto P, Glassberg MK, Lew JI, Elliot SJ. (2018)Estrogen receptor subtype expression and regulation is altered in papillary thyroid cancer after menopause. Surgery. 163(1):143-9.

12. Solbiati L, Volterrani L, Rizzatto G, Bazzocchi M, Busilacci P, Candiani F, Ferrari F, Giuseppetti G, Maresca G, Mirk P. (1985)The thyroid gland with low uptake lesions: evaluation by ultrasound. Radiology. 155(1):187-91.

13. Kolanu BR, Vadakedath S, Boddula V, Kandi V. (2019) Evaluation of the activities of thyroid hormones among pre-and post-menopausal euthyroid women: a cross-sectional study from a tertiary care teaching hospital in India. Cureus. 11(3).
14. Rallison ML, Dobyns BM, Meikle AW, Bishop M, Lyon JL, Stevens W.( 1991) Natural history of thyroid abnormalities: prevalence, incidence, and regression of thyroid diseases in adolescents and young adults. The American journal of medicine. 91(4):363-70.

15. Ceresini G, Milli B, Morganti S, Maggio M, Bacchi-Modena A, Sgarabotto MP, Chirico C, Di Donato P, Campanati P, Valcavi R, Ceda GP. (2008) Effect of estrogen therapy for 1 year on thyroid volume and thyroid nodules in postmenopausal women. Menopause. 15(2):326-31.

16. Sajjadieh HR, Sajjadieh V, Aminorroaya A, Amini M, Oveisgharan S, Reisifar M, Adibi A, Siavash M, Nazem M, Peyman M. (2005) Value of sonography in determining the nature of thyroid nodules: evaluation of the sonographic characteristics. Journal of Diagnostic Medical Sonography. 21(1):38-44. 